\title{
Results of IceCube searches for neutrinos from blazars using seven years of through-going muon data
}

\author{
The IceCube Collaboration ${ }^{\dagger}$ \\ $\dagger$ http://icecube.wisc.edu/collaboration/authors/icrc17_icecube \\ E-mail: mhubereicecube.wisc.edu
}

\begin{abstract}
Located at the South Pole, the IceCube Neutrino Observatory is the world largest neutrino telescope, instrumenting one cubic kilometre of Antarctic ice at a depth between $1450 \mathrm{~m}$ to $2450 \mathrm{~m}$. In 2013 IceCube reported the first observations of a diffuse astrophysical high-energy neutrino flux. Although in the meantime the collaboration has detected more than 50 high energy neutrino events, the origin of these neutrinos is still not identified. Blazars, a subclass of Active Galactic Nuclei and one of the most powerful classes of objects in the universe, have long been considered plausible sources of high energy neutrinos. This scenario can be examined using stacking methods testing the correlation between IceCube neutrinos and catalogs of hypothesized sources. Here the results of stacking analyses using three different blazar catalogs will be presented. The analyses are performed on 7 years of through-going muon data, recorded by IceCube between 2008 and 2015.
\end{abstract}

Corresponding authors: Matthias Huber*1, Kai Krings ${ }^{1}$

${ }^{1}$ Technische Universität München, Physik-Departement, James-Franck-Str. 1, 85748 Garching 


\section{Introduction}

With the first discovery of an astrophysical neutrino flux at PeV energies the IceCube collaboration marked the beginning of a new era of study not only in high energy neutrino astronomy but more generally in the study of the high energy universe [1]. Due to their neutral charge and their very low interaction cross sections neutrinos are neither deflected by magnetic fields nor significantly absorbed in the interstellar medium. Consequently neutrinos carry unique information about their site of production. They are not only ideal messengers for the directional origin of the most powerful objects in the Universe but can also give insight in the acceleration mechanisms within these objects at the highest energies.

Blazars, a subclass of Active Galactic Nuclei (AGNs) hosting a jet of highly relativistic particles pointing towards the Earth, are among the most luminous objects in the entire universe [5]. Depending on the conditions for particle acceleration at the site of these objects, they can be regarded as potential extragalactic sources for the emission of high energy neutrinos [7].

In the following the neutrino signal from three different samples of blazars is tested with a stacking method using seven years of data from the IceCube Neutrino Observatory [2]. The IceCube Observatory is the world's largest neutrino telescope, instrumenting one cubic kilometre of Antarctic ice at a depth between $1450 \mathrm{~m}$ to $2450 \mathrm{~m}$. The detection principle relies on the observation of Cherenkov light emitted from secondary particles created in interactions of neutrinos within the Antarctic ice or the nearby bedrock. Muons, which produce a long luminous track within the detector, are most suitable to get an accurate pointing towards the origin of the events. Above energies of $10 \mathrm{TeV}$ the direction of muon events in IceCube can be reconstructed with a median angular resolution of less than $1^{\circ}$ [3]. Hence the results of this analysis are based on a sample of approximately 700000 through-going muon events from the whole sky integrated over a livetime of seven years.

\section{Blazar populations}

Among the most luminous objects in the universe, blazars generate photons over a broad emission band ranging from radio to $\mathrm{TeV}$ energies. In general the spectral energy distribution (SED) of blazars is composed of two broad humps. While the low energy peak between infrared and x-ray energies can be associated with synchrotron radiation from relativistic electrons, different potential scenarios exist for the generation of the second hump at gamma-ray energies.

Based on different properties in the spectral energy distribution, blazars can be further classified into flat-spectrum radio quasars (FSRQs) and BL Lacertae objects (BL Lacs). While the latter only show weak emission lines in the optical spectrum, strong and broad emission lines are visible in the SED of FSRQs [5]. A second complementary classification is based on the non-thermal emission of blazars. It makes use of the rest frame value of the frequency of the synchrotron peak $v_{\text {peak }}^{S}$, reflecting the maximum energy of the accelerated electrons within the relativistic jets. According to the position of the synchrotron peak blazars are categorized as low and intermediate synchrotron peaked (LSP and ISP) if $v_{\text {peak }}^{S}<10^{15} \mathrm{~Hz}(\sim 4 \mathrm{eV})$ and high synchrotron peaked (HSP) if $v_{\text {peak }}^{S}>10^{15} \mathrm{~Hz}$. In particular HSP BL Lacs (HBL), a rare class of blazars, are very powerful 
gamma-ray emitters. Hence these objects are of special interest in the investigation of the generation processes of ultra high energy cosmic rays and the related high energy neutrinos $[6,7]$.

Following theoretical model explanations, neutrinos could be produced via the decay of charged pions from photo-hadronic interactions of high energy protons with ambient photons or with gas within the jets. This neutrino flux would be accompanied by a flux of very high energy (VHE) gamma-rays from the decay of neutral pions.

The existence of the highest energy IceCube neutrinos $(\sim \mathrm{PeV})$ implies the generation of a $\gamma$-ray flux in the $\geq \mathrm{TeV}$ range [13]. Hence in general blazars with higher gamma-ray flux are assumed to emit more high-energy neutrinos. Yet this conclusion has to be treated carefully. Since VHE gamma-rays are strongly attenuated by extragalactic background light (EBL) or reabsorbed at the source, the photon-neutrino connection on a source by source basis might be diluted. Given that for the theoretical description of the low energy synchrotron hump the existence of relativistic electrons is inevitable, it seems reasonable to assume that also the acceleration of hadrons is possible within the jets of blazars. Nevertheless, it has not yet been possible to determine the fraction of the hadronic component to the high energy hump of blazars nor to disprove purely leptonic models. The detection of neutrinos from blazar populations would directly prove the existence of this hadronic component.

By studying a list of VHE IceCube neutrinos in [7], a possible association between eight HBLs and neutrinos from the tested sample was suggested. In a following study [8], the authors tested the correlation between different blazar catalogs and a list of high energy starting events (HESE) from IceCube, always finding the most significant correlation for the HBLs within each catalog.

Motivated by these observations three different blazar catalogs are analyzed in this paper. These are the second WISE ${ }^{1}$ High Synchrotron Peaked catalog (2WHSP) $[9,10]$, the HBLs from the second catalog of Hard Fermi-LAT ${ }^{2}$ sources (2FHL) [11] and the FSRQs from the third catalog of AGN detected by Fermi-LAT (3LAC) [12]. The locations of the blazars from each of these catalogs are illustrated in Figure 1. Since the goal of this analysis is the detection of extragalactic neutrino point sources only blazars with galactic latitude $\left|b_{I I}\right|>10^{\circ}$ are selected to avoid any bias from the Galactic Plane.

\subsection{WHSP}

The 2WHSP catalog is currently the largest and most complete list of HSP blazars, consisting of 1681 sources [10]. The catalog is built up by correlating data from different multi-wavelength surveys and applying selection criteria based on spectral features in the radio to infra-red (IR) and IR to X-ray region that are known to be specific to blazar SEDs $[9,10]$. Since the selection procedure of the 2WHSP catalog does not rely on gamma-ray observations this sample of blazars is not limited by the sensitivity constraints of currently available gamma-ray surveys. Hence also the effect of attenuation of very high energy photons due to extragalactic background light is negligable. All 2WHSP objects are supposed to radiate up to highest gamma-ray energies. Nevertheless only a few sources are confirmed $\mathrm{GeV}$ or even $\mathrm{TeV}$ photon emitters. To account for this lack of information the 2WHSP catalog provides every source with a quantitative measure of its potential

\footnotetext{
${ }^{1}$ WISE:Wide-field Infrared Survey Explorer

${ }^{2}$ Large Area Telescope
} 


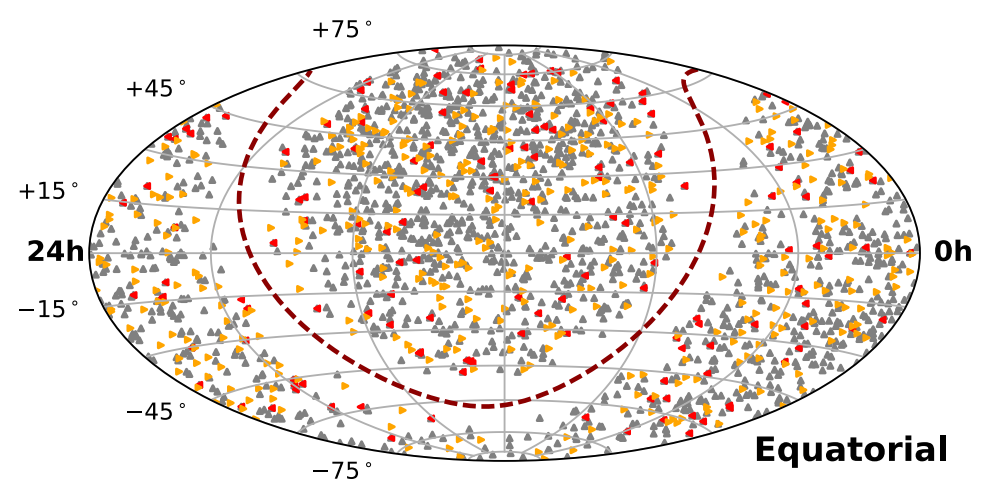

Figure 1: Skymap showing the source positions of the 2WHSP catalog (grey), the 2FHL HBLs (red) and the 3LAC FSRQs (orange). The dotted line (dark red) indicates the location of the galactic plane, with no sources selected in the region with $\left|b_{I I}\right|>10^{\circ}$.

visibility in $\mathrm{TeV}$ range. This property, called figure of merit (FOM), is defined as the ratio of the flux of the synchrotron peak of the blazar in question to the flux of the synchrotron peak of the faintest blazar that was already detected in the $\mathrm{TeV}$ band [9]. Blazars that are powerful enough to generate $\mathrm{TeV}$ gamma-rays might also be potential sources for astrophysical neutrinos.

\subsection{FHL HBLs}

The 2FHL catalog consists of $\sim 360$ extragalactic sources detected by the Fermi LAT during $\sim 6$ years of run time [11]. All sources in this catalog are detected at energies above $50 \mathrm{GeV}$. Since the focus of this paper is only on the connection between blazars and neutrinos, only HBLs are extracted from the catalog yielding a total population of $149 \mathrm{HBLs}$ with galactic latitude $\left|b_{I I}\right|>10^{\circ}$.

\subsection{LAC FSRQs}

Since not only HBLs but also FSRQs are promising candidates for high-energy neutrino emitters, the last blazar sample that is tested in this paper is a population of FSRQs from the 3LAC catalog established by Fermi-LAT [12]. The 3LAC catalog consists of 1591 AGNs detected between $100 \mathrm{MeV}$ and $300 \mathrm{GeV}$, with most of the AGNs being blazars. The subsample of the 3LAC that is used in this analysis consists of 414 confirmed FSRQs at $\left|b_{I I}\right|>10^{\circ}$.

\section{Analysis method}

\subsection{Unbinned likelihood stacking approach}

The through-going muon data set used for this analysis is vastly dominated by track-like events arising from atmospheric muons and neutrinos. Hence in order to find a significant indication of neutrino point sources it is essential to separate any astrophysical neutrino signal from this much larger atmospheric background. The statistical method that is used here to test the connection between the populations of blazars and the IceCube data is based on an unbinned likelihood ratio 
maximization using the likelihood

$$
\mathscr{L}\left(n_{s}, \gamma \mid \boldsymbol{\delta}\right)=\prod_{j}^{N_{\text {sample }}} \prod_{i \in j}\left[\frac{n_{s}^{j}}{N_{\text {tot }}^{j}} \overrightarrow{\mathscr{S}}_{i}^{j}\left(\vec{x}_{i}, \vec{x}_{s}, \sigma_{i}, E_{i}, \gamma\right)+\left(1-\frac{n_{s}^{j}}{N_{\text {tot }}^{j}}\right) \overrightarrow{\mathscr{B}}_{i}^{j}\left(\vec{x}_{s}, E_{i}\right)\right],
$$

with $j$ indexing the samples $j \in\{$ IC40, IC59, IC79, IC86 I-IV $\}$ and $i$ the respective event index. This likelihood is defined as a function of the spectral index $\gamma$ and the number of expected signal events $n_{s}$, where $n_{s}^{j}$ describes the portion of expected signal events coming from sample $j$. The separation power between background and signal likeliness of a specific scenario in Equation (3.1) is mainly based on two event criteria.These criteria are the direction $\vec{x}_{i}$ and the energy $E_{i}$ of the events. Neutrino signal events from point sources are expected to be distributed close to the direction of their origin assuming a Gaussian-distributed spatial signal likelihood $\overrightarrow{\mathscr{S}}_{i}^{j}\left(\vec{x}_{i}, \vec{x}_{s}, \sigma_{i}, E_{i}, \gamma\right)$ for each event position $\vec{x}_{i}$ around the source location $\vec{x}_{s}$ with angular uncertainty $\sigma_{i}$. On the other hand events resulting from atmospheric background are locally spread uniformly in space, in the sense that the frequency of their appearance only depends on the declination angle. Hence the background probability $\overrightarrow{\mathscr{B}}_{i}^{j}\left(\vec{x}_{s}\right)$ for each event $i$ can be obtained from experimental data scrambled in right ascension.

In addition to the event position relative to the source the respective energy information $E_{i}$ can be used to discriminate sources with a $E^{-\gamma}$ spectrum from the atmospheric background, which follows a very soft $E^{-3.7}$ spectrum.

Using the likelihood from Equation (3.1) the test statistic $\Lambda$ is defined as

$$
\Lambda=-2 \log \left[\frac{\mathscr{L}\left(n_{s}=0\right)}{\mathscr{L}\left(n_{s}=\hat{n}_{s}, \gamma=\hat{\gamma}\right)}\right] .
$$

By maximizing this likelihood ratio with respect to the number of expected signal events $\hat{n}_{s}$ and a globally fixed spectral index $\hat{\gamma}$ the validity of the point source hypothesis can be examined.

In case of a stacking analysis not only one point source but multiple point sources are assumed to generate a cumulative astrophysical neutrino signal inside the detector. In order to test such a scenario the signal PDF in Equation (3.1) has to be extended to the more general case of $M$ source candidates

$$
\overrightarrow{\mathscr{S}}_{i} \rightarrow \overrightarrow{\mathscr{S}}_{i}^{\text {Stack }}=\frac{\sum_{k=1}^{M} W^{k} R^{k}\left(\delta_{k}, \gamma\right) \cdot \overrightarrow{\mathscr{S}}_{i}^{j}\left(\vec{x}_{i}, \vec{x}_{s}^{k}, \sigma_{i}, E_{i}, \gamma\right)}{\sum_{k=1}^{M} W^{k} R^{k}\left(\delta_{k}, \gamma\right)}
$$

with $\vec{x}_{s}^{k}=\left(\alpha_{k}, \delta_{k}\right)$ indicating the position of source $k$. This extended signal PDF can be regarded as superposition of the signal PDFs from the individual sources, with each source weighted with a detector specific weight $R^{k}\left(\delta_{k}, \gamma\right)$ accounting for the declination and energy dependent detection efficiency of IceCube and a theoretical weight $W^{k}$ accounting for theoretically predicted parameters of the individual sources.

\subsection{Catalog scans}

For the statistical analysis method in this paper all blazars in the respective catalog are assumed to emit neutrinos with the same strength, yielding $W^{k}=\frac{1}{M}$. Nevertheless for all three catalogs in this paper a correlation between the VHE gamma-ray flux and the neutrino flux is taken into 
Table 1: Pre trial-corrected results of the 10 cumulative subsamples of the 2 WHSP catalog.

\begin{tabular}{c||cccccccccc}
\hline \hline \# Sources & 10 & 17 & 26 & 38 & 60 & 136 & 252 & 516 & 1099 & 1681 \\
\hline FOM $(\geq)$ & 4.00 & 4.00 & 2.55 & 2.00 & 1.60 & 1.00 & 0.50 & 0.25 & 0.10 & 0.00 \\
$\gamma$ & 3.2 & 3.3 & 3.2 & 3.1 & 3.2 & 3.2 & 3.0 & 2.7 & 2.7 & 2.6 \\
p-value & 0.013 & 0.212 & 0.341 & 0.161 & 0.236 & 0.432 & 0.531 & 0.058 & 0.065 & 0.224 \\
\hline \hline
\end{tabular}

account indirectly on the whole sample scale. The two Fermi-LAT catalogs are partitioned into cumulative subsets, starting with the subset of blazars with the highest integrated gamma-ray fluxes and adding up this set with blazars having the next higher fluxes. Since the individual sources in each subsample are still weighted equally within the stacking method $\left(W^{k}=\frac{1}{M_{\text {sub }}}\right.$, with $M_{\text {sub }}$ being the number of blazars in the respective subsample), the analyses do not strongly depend on the correlation assumption. Since for the 2WHSP catalog no complete gamma-ray observations are available, the FOM (refer to section 2.1) is used instead of the flux for the partitioning. The exact partitioning is chosen according to the binning in [8].

For each of the subsamples of each catalog the $\mathrm{p}$-value is evaluated. The final post-trial p-value of each catalog is then the trial corrected best $\mathrm{p}$-value from the respective subsamples, yielding in total three final p-values from this analysis.

\section{Results}

None of the three blazar catalogs tested showed any significant evidence for a neutrino signal above background expectations. The pre-trial corrected results of all catalogs are listed in Table 1 and 2. For the 2WHSP catalog the best pre-trial p-value results from the subsample including the 10 blazars with the highest FOM values in the catalog, yielding a post-trial p-value of $7.6 \%$.

Scanning the 2FHL HBL sample yields a most significant pre-trial p-value of $8.7 \%$ arising from the subsample including 127 blazars. The final post-trial p-value of the 2FHL HBL catalog is $24.8 \%$. Similar to the 2WHSP catalog the best pre-trial p-value of FSRQ population from the 3LAC sample is received from smallest subsample, yielding a post-trial p-value of $46 \%$.

All the outcomes from the three catalog stacking analyses are fully compatible with background fluctuations. Moreover looking at the catalog scans (shown in Tables 1 and 2) there is no obvious correlation visible between the integrated gamma-ray flux (or FOM respectively) and the neutrino p-values.

\section{Conclusions}

Since no significant evidence for neutrino emission from the blazar samples analyzed was found, upper limits on the $v_{\mu}$ flux from these blazar samples were calculated. The astrophysical muon neutrino flux observed by the IceCube collaboration yields a spectrum compatible with a single unbroken power law and spectral index of $\gamma=2.13 \pm 0.13$ between $194 \mathrm{TeV}$ and $7.8 \mathrm{PeV}$ [4]. Using a similar power law for the blazar sample, the maximum contribution from that population

\footnotetext{
${ }^{3}$ Overlaps of the catalogs are not taken into account in the trial correction.
} 
Table 2: Pre-trial corrected results of the 2FHL HBL (left) and 3LAC FSRQ catalog (right). For the 2 FHL HBL catalog the flux parameter corresponds to the integrated gamma-ray flux above $50 \mathrm{GeV}$. For the 3LAC FSRQs the flux corresponds to the integrated flux above $100 \mathrm{MeV}$.

\begin{tabular}{|c|c|c|c|c|c|c|c|}
\hline \# Sources & flux $_{2 \mathrm{FHL}}$ & $\gamma$ & p-value & \# Sources & flux $_{3 \mathrm{LAC}}$ & $\gamma$ & p-value \\
\hline 12 & $\geq 10^{-10.00}$ & 3.1 & 0.103 & 13 & $\geq 1.0 \cdot 10^{-8}$ & 2.3 & 0.148 \\
\hline 36 & $\geq 10^{-10.45}$ & 4.0 & $>0.66$ & 20 & $\geq 7.0 \cdot 10^{-9}$ & 2.4 & 0.352 \\
\hline 42 & $\geq 10^{-10.50}$ & 3.5 & 0.620 & 29 & $\geq 5.6 \cdot 10^{-9}$ & 2.4 & 0.454 \\
\hline 61 & $\geq 10^{-10.60}$ & 3.4 & 0.500 & 40 & $\geq 4.9 \cdot 10^{-9}$ & 2.8 & 0.205 \\
\hline 92 & $\geq 10^{-10.75}$ & 2.4 & 0.149 & 59 & $\geq 3.1 \cdot 10^{-9}$ & 4.0 & $>0.5$ \\
\hline 127 & $\geq 10^{-10.90}$ & 3.0 & 0.087 & 102 & $\geq 1.9 \cdot 10^{-9}$ & 2.9 & 0.532 \\
\hline 143 & $\geq 10^{-11.00}$ & 3.1 & 0.092 & 172 & $\geq 1.0 \cdot 10^{-9}$ & 3.1 & 0.222 \\
\hline \multirow[t]{2}{*}{149} & $\geq 10^{-11.13}$ & 3.0 & 0.289 & 353 & $\geq 3.1 \cdot 10^{-10}$ & 3.0 & 0.256 \\
\hline & & & & 414 & $\geq 1.0 \cdot 10^{-10}$ & 3.0 & 0.290 \\
\hline
\end{tabular}

to the diffuse flux can be evaluated. The $90 \%$ C.L. upper limit for the 2FHL HBLs following a $\gamma=2.13$ power law is illustrated in Figure 2. Assuming this unbroken power law the 2FHL HBLs can explain at most $4.5 \%$ to $5.7 \%$ of the diffuse flux at $90 \%$ C.L.. Instead, when assuming that the neutrino emission strength of the individual blazars is directly proportional to their gamma-ray flux, the 2FHL HBLs can only describe $3.8 \%$ of the diffuse $v_{\mu}$ flux.

Since there is no indication for the blazar populations to follow a single unbroken power law, other scenarios are also tested. Several theoretical models allow blazars to follow a very hard spectrum [14]. In case of such a hard spectrum an energy cut off is expected once the hadronic acceleration of protons at the source breaks off. In the right part of Figure 2 the flux upper limits for $\gamma=1.5$ and $\gamma=1.0$ with different cut off energies are shown. In case of a spectral index $\gamma=1.5$ with an exponential cut off at $1 \mathrm{PeV}$, the $2 \mathrm{FHL}$ HBLs can contribute at most $\sim 12 \%$ to the diffuse muon neutrino flux between $0.2 \mathrm{PeV}$ and $1.2 \mathrm{PeV}$. When assuming an even harder spectral index of $\gamma=1.0$ with the same cut off at $1 \mathrm{PeV}$, the $2 \mathrm{FHL}$ HBLs might still be able to explain up to $\sim 21 \%$ of the diffuse flux between $0.4 \mathrm{PeV}$ and $1.7 \mathrm{PeV}$.

In [14] the shape of the SED of several HBLs was modeled with a leptohadronic emission model, automatically yielding a prediction for the neutrino emission. For Mrk 421 the prediction was found to be in good agreement with the neutrino flux implied by a cascade-like IceCube event (ID 9) located in the vicinity of Mrk 421[14, 7]. Using this neutrino emission shape from Mrk 421 for all of the 2FHL HBLs, this blazar population could still account for no more than $\sim 27 \%$ of the diffuse flux within $1.3 \mathrm{PeV}$ and $3.8 \mathrm{PeV}$.

Consequently one can find that the population of the 149 HBLs from the $2 \mathrm{FHL}$ catalog does not account for the majority of the diffuse muon neutrino flux at $90 \%$ C.L.. Nevertheless depending on the spectral shape of the neutrino emission of the blazar sample they could still explain up to $27 \%$ of the diffuse flux within certain energy ranges.

Adding more IceCube data to this analysis will improve our sensitivity towards a significant signal detection in the next years. Nevertheless assuming for instance that the actual neutrino flux of the 2FHL HBLs corresponds to the upper limit flux of the model from [14] one would still need 

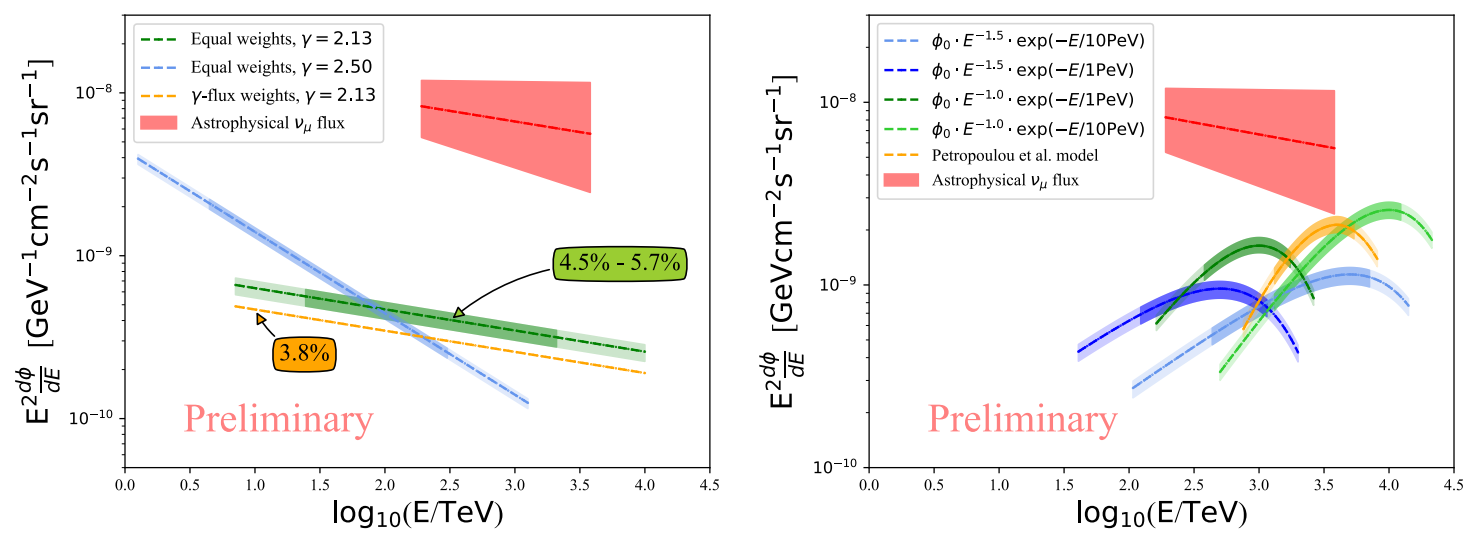

Figure 2: $90 \%$ C.L. upper limits of the 2FHL HBL catalog using different assumptions on the spectral shape of the blazars. All fluxes are given in intensity units. The dark-shaded bands illustrate the $1 \sigma$ energy regions where IceCube has the highest exclusion power for the particular model, the light shaded bands the $90 \%$ region. The best fit to the astrophysical diffuse muon neutrino flux $\phi_{v_{\mu}+\bar{v}_{\mu}}^{\text {astro }}$ from [4] including the $68 \%$ C.L. contours is displayed in red. Left: Equal and gamma-ray flux weighted limits for single unbroken power laws. The numbers illustrate the maximal contribution to the diffuse $v_{\mu}$-flux. Right: Limits for single power laws with different energy cut offs and the limit using the theoretical model from [14].

at least $\sim 10$ more years of data from the current IceCube detector to get a $5 \sigma$ discovery. Hence in the future, the analysis can also be extended to larger HBL samples, such as 3FHL catalog, being an extension of the 2FHL catalog.

\section{References}

[1] IceCube Collaboration, M. Aartsen et al., Science 342 (2013) 1242856.

[2] IceCube Collaboration, M. Aartsen et al., JINST 12 (2017) P03012.

[3] IceCube Collaboration, M. Aartsen et al., Astrophys. J. 835 (2017) 2.

[4] IceCube Collaboration, M. Aartsen et al., Astrophys. J. 833 (2016) 1.

[5] C. Urry and P. Padovani, Publ. Astron. Soc. Pac. 107 (1995).

[6] P. Padovani et al., Astrophys. J. 444 (1995).

[7] P. Padovani et al., Mon. Not. Roy. Astron. Soc. 443 (2014) 1.

[8] P. Padovani et al., Mon. Not. Roy. Astron. Soc. 457 (2016) 4.

[9] B. Arsioli et al., Astron. Astrophys. 579 (2015) A34.

[10] B. Arsioli et al., Astron. Astrophys. 598 (2017) A17.

[11] Fermi-LAT Collaboration, M. Ackermann et al., Astrophys. J. Suppl. 222 (2016) 1.

[12] Fermi-LAT Collaboration, M. Ackermann et al., Astrophys. J. 810 (2015) 1.

[13] Kelner, S. R. et al., Phys. Rev. D78 (2008) .

[14] M. Petropoulou et al., Mon. Not. Roy. Astron. Soc. 448 (2015) 3, 2412-2429. 